University of Texas at El Paso

ScholarWorks@UTEP

$1-2017$

\title{
Why Unexpectedly Positive Experiences Make Decision Makers More Optimistic: An Explanation
}

Andrzej Pownuk

The University of Texas at El Paso, ampownuk@utep.edu

Vladik Kreinovich

The University of Texas at El Paso, vladik@utep.edu

Follow this and additional works at: https://scholarworks.utep.edu/cs_techrep

Part of the Computer Sciences Commons

Comments:

Technical Report: UTEP-CS-17-01

To appear in Proceedings of the 10th International Workshop on Constraint Programming and Decision Making CoProd'2017, El Paso, Texas, November 3, 2017.

\section{Recommended Citation}

Pownuk, Andrzej and Kreinovich, Vladik, "Why Unexpectedly Positive Experiences Make Decision Makers More Optimistic: An Explanation" (2017). Departmental Technical Reports (CS). 1117.

https://scholarworks.utep.edu/cs_techrep/1117

This Article is brought to you for free and open access by the Computer Science at ScholarWorks@UTEP. It has been accepted for inclusion in Departmental Technical Reports (CS) by an authorized administrator of ScholarWorks@UTEP. For more information, please contact Iweber@utep.edu. 


\title{
Why Unexpectedly Positive Experiences Make Decision Makers More Optimistic: An Explanation
}

\author{
Andrzej Pownuk and Vladik Kreinovich \\ Computational Science Program \\ University of Texas at El Paso \\ 500 W. University \\ El Paso, TX 79968, USA \\ ampownuk@utep.edu,vladik@utep.edu
}

\begin{abstract}
Experiments show that unexpectedly positive experiences make decision makers more optimistic. However, there seems to be no convincing explanation for this experimental fact. In this paper, we show that this experimental phenomenon can be naturally explained within the traditional utility-based decision theory.
\end{abstract}

\section{Formulation of the Problem}

Experimental phenomenon. Experiments show that unexpectedly positive experiences make decision makers more optimistic. This was first observed on an experiment with rats [10]: rats like being ticked, and tickled rats became more optimistic. Several later papers showed that the same phenomenon holds for other decision making situations as well; see, e.g., [2, 7].

Similarly, decision makers who had an unexpectedly negative experiences became more pessimistic; see, e.g., [8].

Why: a problem. There seems to be no convincing explanation for this experimental fact.

What we do in this paper. In this paper, we show that this experimental phenomenon can be naturally explained within the traditional utility-based decision theory.

\section{Formulating the Problem in Precise Terms}

In precise terms, what does it mean to becomes more optimistic or less optimistic? The traditional utility-based decision theory describes the 
behavior of a rational decision maker in situations in which we know the probabilities of all possible consequences of each action; see, e.g., [1, 5, 6, 9]. This theory shows that under this rationality assumption, preferences of a decision maker can be described by a special function $U(x)$ called utility function, so that a rational decision maker selects an alternative $a$ that maximizes the expected value $u(a)$ of the utility.

In this case, there is no such thing as optimism or pessimism: we just select the alternative which we know is the best for us.

The original theory describes the behavior of decision makers in situations in which we know the probability of each possible consequence of each action. In practice, we often have only partial information about these probabilities - and sometimes, no information at all. In such situations, there are several possible probability distributions consistent with our knowledge. For different distributions, we have, in general, different values of the expected utility. As a result, for each alternative, instead of the exact value of the expected utility, we have an interval $[\underline{u}(a), \bar{u}(a)]$ of possible values of $u(a)$. How can we make a decision based on such intervals?

In this case, natural rationality ideas lead to the conclusion that a decision should select an alternative $a$ for which, for some real numbers $\alpha \in[0,1]$, the combination $u(a)=\alpha \cdot \bar{u}(a)+(1-\alpha) \cdot \underline{u}(a)$ is the largest possible; see, e.g., [4]. This idea was first proposed by the Nobelist Leo Hurwicz in [3].

The selection of $\alpha$, however, depends on the person. The value $\alpha=1$ means that the decision maker only takes into account the best possible consequences, and completely ignores possible less favorable situations. In other words, the values $\alpha=1$ corresponds to complete optimism.

Similarly, the value $\alpha=0$ means that the decision maker only takes into account the worst possible consequences, and completely ignores possible more favorable situations. In other words, the values $\alpha=0$ corresponds to complete pessimism.

Intermediate values $\alpha$ mean that we take into account both positive and negative possibilities. The larger $\alpha$, the close this decision maker to complete optimism. The smaller $\alpha$, the closer the decision maker to complete pessimism. Because of this, the parameter $\alpha$ - known as the optimism-pessimism index-is a numerical measure of the decision maker's optimism.

In these terms:

- becoming more optimistic means that the value $\alpha$ increases, and

- becoming less optimistic means that the value $\alpha$ increases.

Thus, the above experimental fact takes the following precise meaning:

- if a decision maker has unexpectedly positive experiences, then this decision maker's $\alpha$ increases;

- if a decision maker has unexpectedly negative experiences, then this decision maker's $\alpha$ decreases.

This is the phenomenon that we need to explain. 


\section{Towards the Desired Explanation}

Optimism-pessimism parameter $\alpha$ can be naturally interpreted as the subjective probability of positive outcome. The value $\alpha$ means that the decision maker selects an alternative $a$ for which the value $\alpha \cdot \bar{u}(a)+(1-\alpha) \cdot \underline{u}(a)$ is the largest possible.

Here, the value $\bar{u}(a)$ corresponds to the positive outcome, and the value $\underline{u}(a)$ corresponds to negative outcome.

For simplicity, let us consider the situation when we have only two possible outcomes:

- the positive outcome, with utility $\bar{u}(a)$, and

- the negative outcome, with utility $\underline{u}(a)$.

A traditional approach to decision making, as we have mentioned, assumes that we know the probabilities of different outcomes. In this case of uncertainty, we do not know the actual (objective) probabilities, but we can always come up with estimated (subjective) ones.

Let us denote the subjective probability of the positive outcome by $p_{+}$. Then, the subjective probability of the negative outcome is equal to $1-p_{+}$. The expected utility is equal to $p_{+} \cdot \bar{u}(a)+\left(1-p_{+}\right) \cdot \underline{u}(a)$.

This is exactly what we optimize when we use Hurwicz's approach, with $\alpha=p_{+}$. Thus, the value $\alpha$ can be interpreted as the subjective probability of the positive outcome.

A new reformulation of our problem. In these terms, the above experimental phenomenon takes the following form:

- unexpectedly positive experiences increase the subjective probability of a positive outcome, while

- unexpectedly negative experiences decrease the subjective probability of a positive outcome.

To explain this phenomenon, let us recall where subjective probabilities come from.

Where subjective probabilities come from? A natural way to estimate the probability of an event is to consider all situations in which this event could potentially happen, and then take the frequency of this event - i.e., the ratio $n / N$ of the number of times $n$ when it happens to the overall number $N$ of cases - as the desired estimate for the subjective probability. For example, if we flip a coin 10 times and it fell heads 6 times out of 10, we estimate the probability of the coin falling heads as 6/10.

Let us show that this leads to the desired explanation.

Resulting explanation. Suppose that a decision maker had $n$ positive experiences in the past $N$ situations. Then, the decision maker's subjective probability of a positive outcome is $p_{+}=n / N$. 
Unexpectedly positive experiences means that we have a series of new experiments, in which the fraction of positive outcomes was higher than the expected frequency $p_{+}$. In other words, unexpectedly positive experiences means that $n^{\prime} / N^{\prime}>p$, where $N^{\prime}$ is the overall number of new experiences, and $n^{\prime}$ is the number of those new experiences in which the outcome turned out to be positive.

How will these new experiences change the decision maker's subjective probability? Now, the decision maker has encountered overall $N+N^{\prime}$ situations, of which $n+n^{\prime}$ were positive. Thus, the new subjective probability $p_{+}^{\prime}$ is equal to the new ratio $p_{+}^{\prime}=\frac{n+n^{\prime}}{N+N^{\prime}}$. Here, by definition of $p_{+}$, we have

$$
n=p_{+} \cdot N
$$

and, due to unexpected positiveness of new experiences, we have $n^{\prime}>p_{+} \cdot N^{\prime}$. By adding this inequality and the previous equality, we conclude that $n+n^{\prime}>$ $p_{+} \cdot\left(N+N^{\prime}\right)$, i.e., that

$$
p_{+}^{\prime}=\frac{n+n^{\prime}}{N+N^{\prime}}>p_{+} .
$$

In other words, unexpectedly positive experiences increase the subjective probability of a positive outcome.

As we have mentioned, the subjective probability of the positive outcome is exactly the optimism-pessimism coefficient $\alpha$. Thus:

- the original subjective probability $p_{+}$is equal to the original optimismpessimism coefficient $\alpha$, and

- the new subjective probability $p_{+}^{\prime}$ is equal to the new optimism-pessimism coefficient $\alpha^{\prime}$.

So, the inequality $p_{+}^{\prime}>p_{+}$means that $\alpha^{\prime}>\alpha$, i.e., that unexpectedly positive experiences make the decision maker more optimistic. This is exactly what we wanted to explain.

Similarly, if we had unexpectedly negative experiences, i.e., if we had $n^{\prime}<$ $p_{+} \cdot N^{\prime}$, then we similarly get $n+n^{\prime}<p_{+} \cdot\left(N+N^{\prime}\right)$ and thus,

$$
p_{+}^{\prime}=\frac{n+n^{\prime}}{N+N^{\prime}}<p_{+}
$$

and $\alpha^{\prime}<\alpha$. So, we conclude that unexpectedly negative experiences make the decision maker less optimistic. This is also exactly what we observe. So, we have the desired explanation.

\section{Acknowledgments}

This work was supported in part by the National Science Foundation grants HRD-0734825 and HRD-1242122 (Cyber-ShARE Center of Excellence) and DUE-0926721, and by an award "UTEP and Prudential Actuarial Science Academy and Pipeline Initiative" from Prudential Foundation. 


\section{References}

[1] P. C. Fishburn, Utility Theory for Decision Making, John Wiley \& Sons Inc., New York, 1969.

[2] C. A. Hales, S. A. Stuart, M. H. Anderson, E. S. J. Robinson, "Modelling cognitive affective biases in major depressive disorder using rodents", British Journal of Pharmacology, 2014, Vol. 171, No. 20, pp. 4524-4538.

[3] L. Hurwicz, Optimality Criteria for Decision Making Under Ignorance, Cowles Commission Discussion Paper, Statistics, No. 370, 1951.

[4] V. Kreinovich, "Decision making under interval uncertainty (and beyond)", In: P. Guo and W. Pedrycz (eds.), Human-Centric Decision-Making Models for Social Sciences, Springer Verlag, 2014, pp. 163-193.

[5] R. D. Luce and R. Raiffa, Games and Decisions: Introduction and Critical Survey, Dover, New York, 1989.

[6] H. T. Nguyen, O. Kosheleva, and V. Kreinovich, "Decision making beyond Arrow's 'impossibility theorem', with the analysis of effects of collusion and mutual attraction", International Journal of Intelligent Systems, 2009, Vol. 24, No. 1, pp. 27-47.

[7] J. Panksepp, J. S. Wright, M. D. Döbrössy, Th. E. Schlaepfer, and V. A. Coenen, "Affective neuroscience strategies for understanding and treating depression: from preclinical models to three novel therapeutics", Clinical Psychological Science, 2014, Vol. 2, No. 4, pp. 472-494.

[8] J. Papciak, P. Popik, E. Fuchs, R. Rygula, "Chronic psychosocial stress makes rats more 'pessimistic' in the ambiguous-cue interpretation paradigm", Behavioural Brain Research, 2013, Vol. 256, pp. 305-310.

[9] H. Raiffa, Decision Analysis, Addison-Wesley, Reading, Massachusetts, 1970.

[10] R. Rygula, H. Pluta, and P. Popik, "Laughing rats are optimistic", PLoS ONE, 2012, Vol. 7, No. 2, Paper e51959. 